\title{
STRUCTURAL STUDY OF A NEW 1-(2-THIENYL)-3-(3-METHYL-2-THIENYL)-PROPENONE, IN-SITU RADIATION-INDUCED DIMERIZATION PRODUCTS
}

\author{
Y. MORENO ${ }^{\prime *}$, D. CONTRERAS ${ }^{l}$, A. BULJAN ${ }^{2}, F . B R O V E L L I^{3}$, R. BAGGIO \\ 1 Depto. de Quím. Analítica e Inorgánica, Fac. Cs. Químicas, Universidad de Concepción, Chile. \\ 2_Depto. de Fisicoquímica (Lab. QTC), Fac. Cs. Químicas, Universidad de Concepción, Chile. \\ 3 Depto. de Cs. Básicas, Campus Los Ángeles, Universidad de Concepción, Chile, \\ 4_Departamento de Física, Comisión Nacional de Energía Atómica, Buenos Aires, Argentina.
}

(Received: September 1, 2010 - Accepted: December 28, 2010)

\begin{abstract}
The new conjugated system (“chalcone kind”) reported herein, 1-(2-thienyl)-3-(3-methyl-2-thienyl)-propenone, $\mathrm{C}_{12} \mathrm{H}_{10} \mathrm{OS}_{2}$ presents a monomeric molecular structure, and it most interesting aspects are to be found in it intermolecular interactions, of varied type $(\mathrm{C}-\mathrm{H} \cdots \mathrm{O}, \mathrm{C}-\mathrm{H} \cdots \pi, \pi \cdots \pi)$ and strength. Some anomalies detected in the refinement process, however, provided evidence that an unexpected in-situ, radiation-induced dimerization reaction had taken place during data collection, with the starting and final products coexisting in a topotactic fashion. This effect is depended of structural packing; that is not all this kind of conjugated system presents this effect. The main factor is the distance between the double bonds of neighboring monomers (about $3 \AA$ )
\end{abstract}

\section{INTRODUCTION}

The electronic and optoelectronic applications of conjugated polymers as organic wide-gap semi-conductors have attracted considerable interest in the last decade. In this field, polythiophenes derivatives are among the most studied because of their potential use in organic light-emitting diodes. ${ }^{1-3}$ These polymers (used to prepare electroluminicent devices) are obtained through monomer polymerization via electrochemical deposition on indium tin oxide.

The polymeric precursors employed have been a series of heterocyclic $\alpha$, $\beta$-unsaturated ketones containing thiophene rings. $2,4,5$ similar to the molecule reported in this work and they have been studied and characterized by electrochemical and ab-initio methods. ${ }^{4,6,9}$

In general there is not much structural information about this class the compounds. The structural information available is related to conformational studies and crystal structure as electronic interactions between oxygen of carbonyl group and the heteroatom ${ }^{5,7,9}$ and interatomic interactions of type C-H...O, C-H... $\pi, \ldots \pi^{10,11}$

Following our interest in this kind of compounds, mainly directed to their use as potential ligands in further complex development we have synthesized and characterized a new compound in this populous family, viz: 1-(2-thienyl)3-(3-methyl-2-thienyl)-propenone, $\mathrm{C}_{12} \mathrm{H}_{10} \mathrm{OS}_{2}$. See scheme 1.

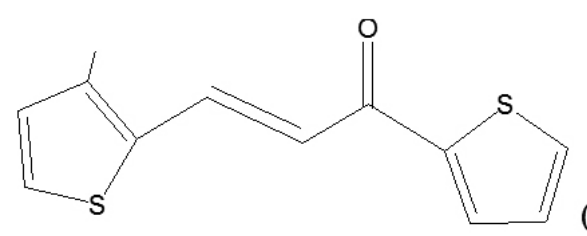

In this work, we report the crystal structure of 1-(2-thienyl)-3-(3-methyl2-thienyl)-propenone. The structure was solved by single crystal X-ray diffraction.

\section{EXPERIMENTAL}

\section{SYNTHESIS}

Compound $\mathrm{C}_{12} \mathrm{H}_{40} \mathrm{OS}_{2}$ was synthesized following methods reported in literature. 4, 10, 11 3-methyl-2-thiophenecarboxaldehyde $(0.1 \mathrm{~mol})$ was mixed with 2-acetylthiophene $(0.1 \mathrm{~mol})$. The mixture was cooled at $0^{\circ} \mathrm{C}$ and $\mathrm{KOH} /$ ethanol $20 \%$ solution was slowly added. The mixture was stirred during 12 $\mathrm{h}$. Then the mixture was filtered and washed with cool ethanol. The product was recrystallized from an ethanol/water solution (5:1) and pale yellow crystals were obtained. The yield was $70 \%$. The elemental analysis gives the following results: (\%) (exp/theo): 61.47/61.50 (C); 4.27/4.30 (H); 6.78/6.83 (O); 27.18/27.37 (S).

\section{IR SPECTRUM}

Spectra of 1-(2-thienyl)-3-(3-methyl-2-thienyl)-propenone in KBr pellets were recorded on a FTIR-NICOLET MAGNA 550 spectrometer.

\section{X-RAY DIFFRACTION}

Diffraction spectrums were record in a Bruker SMART AXS CCD diffractometer with graphite-monochromated Mo $K a$ radiation $(1=0.71073$ $\AA$ ). Semi-empirical absorption correction based on symmetry equivalent reflections was applied. A total of 8994 reflections were collected, of which 3877 reflections were unique (Rint $=0.057$ ). The structure was resolved by direct methods and refined by the full-matrix least-squares method based on F2. Structure solution, refinement, and generation of publication materials were performed with the use of the SHELXTL crystallographic software package ${ }^{12-15}$. The final refinement includes anisotropic displacement parameters for the non-hydrogen atoms. The crystal data and structure refinement of Compound $\mathrm{C}_{12} \mathrm{H}_{10} \mathrm{OS}_{2}$ were summarized in Table 1.

\section{REFINEMENT}

Table 1-3 shows the crystal data and refinement details. The principal problems in this compound was the coexistence of symmetry related monomeric units and their cyclation dimerization products resulting from irradiation. The transformations were clearly topotactic, but resented the crystal and (accordingly) the refinement quality. The amount of dimerized material was directly related to the more or less favorable position in the original packing for such a reaction (see comment section for a detailed discussion). Hydrogen atoms were placed at calculated positions (C-H: 0.93-0.97 $\AA$ ) and allowed to ride; methyl groups were allowed to rotate as well. Displacement factors were taken as $\mathrm{U}(\mathrm{H})$ isot $=\mathrm{x} . \mathrm{U}($ host $), \mathrm{x}: 1.2-1.5$ 
Table 1, Crystal data.

\begin{tabular}{|l|l|}
\hline $\mathrm{C}_{12} \mathrm{H}_{10} \mathrm{O}_{1} \mathrm{~S}_{2}$ & $\mathrm{~V}=1112.2(4) \AA^{3}$ \\
\hline $\mathrm{Mr}=234.32$ & $\mathrm{Z}=4$ \\
\hline Triclinic, $\mathrm{P}^{-} 1$ & $\mathrm{Dx}=1.399 \mathrm{Mg} \mathrm{m}^{-3}$ \\
\hline $\mathrm{a}=9.671(2) \AA$ & $\mathrm{Mo} \mathrm{K} \alpha$ \\
\hline $\mathrm{b}=9.689(2) \AA$ & $\mu=0.45 \mathrm{~mm}^{-1}$ \\
\hline $\mathrm{c}=13.772(3) \AA$ & $\mathrm{T}=100(2) \mathrm{K}$ \\
\hline$\alpha=74.381(7)^{\circ}$ & Prism, yellow \\
\hline$\beta=74.854(7)^{\circ}$ & $0.19 \times 0.11 \times 0.08 \mathrm{~mm}$ \\
\hline$\gamma=65.242(6)^{\circ}$ & \\
\hline
\end{tabular}

\begin{tabular}{|l|l|}
\hline SMART Bruker-AXS diffractometer & 3877 independent reflections \\
\hline CCD rotation images, thin slices scans & 2943 reflections with $I>2 \sigma(I)$ \\
\hline $\begin{array}{l}\text { Absorption correction: } \text { multi- } \\
\text { scanSADABS }{ }^{15}\end{array}$ & Rint $=0.057$ \\
\hline Tmin $=0.93$, Tmax $=0.97$ & $\theta \max =25.0^{\circ}$ \\
\hline 8994 measured reflections & \\
\hline
\end{tabular}

Table 3: Refinement

\begin{tabular}{|l|l|}
\hline Refinement on $\mathrm{F}^{2}$ & H-atom parameters constrained \\
\hline $\mathrm{R}\left[\mathrm{F}^{2}>2 \sigma\left(\mathrm{F}^{2}\right)\right]=0.071$ & $\begin{array}{l}\mathrm{W}=1 /\left[\sigma^{2}\left(\mathrm{Fo}^{2}\right)+(0.023 \mathrm{P})^{2}+\right. \\
2.9 \mathrm{P}] \text { where } \mathrm{P}=\left(\mathrm{F}_{\mathrm{o}}^{2}+2 \mathrm{~F}_{\mathrm{c}}{ }^{2}\right) / 3\end{array}$ \\
\hline $\mathrm{wR}\left(\mathrm{F}^{2}\right)=0.143$ & $(\Delta / \sigma)_{\max }<0.001$ \\
\hline $\mathrm{S}=1.09$ & $\Delta \rho \max =0.97 \mathrm{e} \AA^{-3}$ \\
\hline 3877 reflections & $\Delta \rho \min =-0.70 \mathrm{e} \AA^{-3}$ \\
\hline 287 parameters & Extinction correction: none \\
\hline
\end{tabular}

\section{RESULT AND DISCUSSION}

\section{STRUCTURAL STUDY}

Compounds $\mathrm{C}_{12} \mathrm{H}_{10} \mathrm{O}_{2} \mathrm{~S}$ present two independent molecules in the asymmetric unit (Table 1-3 shows the crystal data and refinement details). We shall describe in what follows the monomeric part, drawn in shaded full thermal ellipsoids and full lines in Figures 1. The dimerization products (drawn in open ellipsoids and bonds, Fig. 1b) will be treated afterwards.
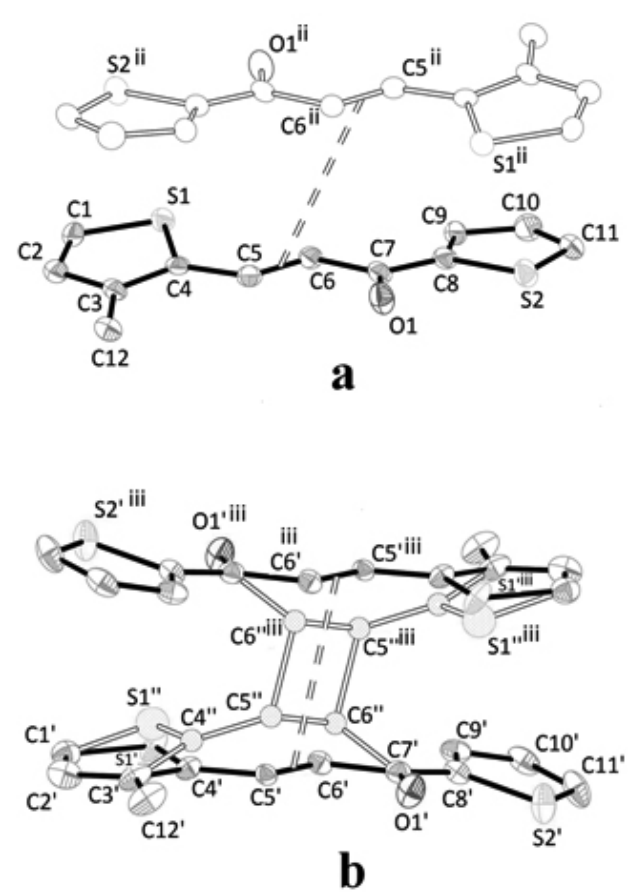

Fig.1: Molecular diagram of $\mathrm{C}_{12} \mathrm{H}_{10} \mathrm{O}_{2} \mathrm{~S}$. Anisotropic displacement ellipsoids drawn at a $40 \%$ level. a) The unprimed, non-dimerized molecule. b) The primed moiety, showing in hollow bonds, the dimerized part. The doubly primed indicates the displacement of the electronic density owing to the radiation. Symm. op.: ii) 2-x, 1-y, -z iii)-x,-y,-z

The two independent molecules present the same conformation; that is two terminal thiophene rings bridged by a propa-1-en-3-one link, with both rings almost coplanar to the whole group and with the sulfur atoms in trans conformation. The oxygen in the carbonyl group is cis to the nearest sulfur atom and trans to the other one

The planarity of the group is perturbed by a slight twist along the bridge axis, difficult to quantify due to the disordered nature of the groups. In general, the spatial arrangement is governed by a number of intermolecular interactions, of varied type and strength.

The intermolecular interactions (mainly of the $\mathrm{C}-\mathrm{H} \cdots \mathrm{O}$ type, Table 4) do not "mix" unrelated molecules ("primed" and "unprimed" ones in Fig. 1); they generate, instead, two distinct families of non intersecting 1D structures parallel to [100] and [010] as shown in Fig 2

Tables 5 and 6 show some distances and angles respectively

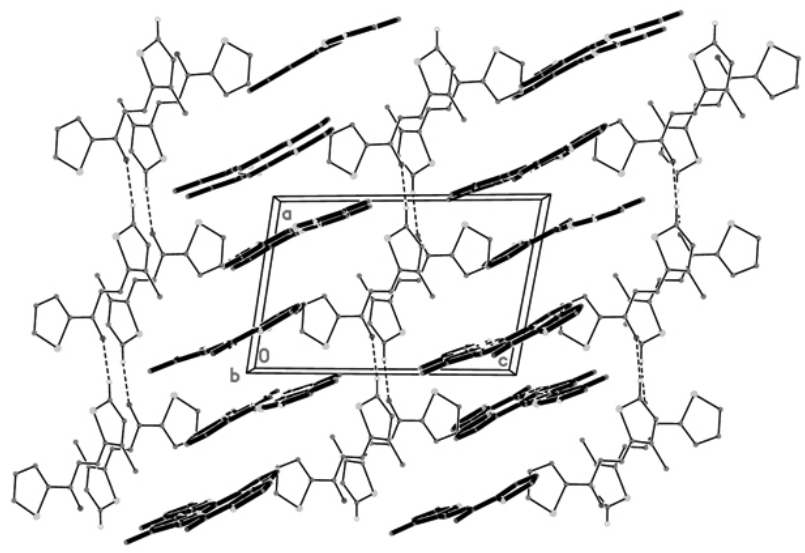

Fig.2: Packing diagram of $\mathrm{C}_{12} \mathrm{H}_{10} \mathrm{O}_{2} \mathrm{~S}$, showing in bold lines the unprimed molecules (forming chains coming out of the figure) and primed ones, in thin lines (as vertical chains). 
Table 4: Hydrogen-bond geometry $\left(\AA,^{\circ}\right)$.

\begin{tabular}{|c|c|c|c|c|}
\hline$D-\mathrm{H}^{\prime} \cdots A$ & $D-\mathrm{H}$ & $\mathrm{H} \cdots A$ & $D \cdots A$ & $D-\mathrm{H}^{\cdots} A$ \\
\hline $\mathrm{C} 1^{\prime}-\mathrm{H} 1^{\prime} \cdots \mathrm{O} 1^{\mathrm{i}}$ & 0.95 & 2.33 & $3.279(6)$ & 167 \\
\hline $\mathrm{C} 1-\mathrm{H} 1^{\cdots} \cdots 1^{\mathrm{ii}}$ & 0.95 & 2.29 & $3.220(6)$ & 165 \\
\hline
\end{tabular}

Symmetry codes: (i) $x+1, y, z$; (ii) $x, y+1, z$.

Table 5: Some important distances $(\AA)$ of $\mathrm{C} 12 \mathrm{H} 10 \mathrm{O} 2 \mathrm{~S}$.

\begin{tabular}{|c|c|}
\hline bond & distance/ $\AA$ \\
\hline C4-C5 & $1.43(1)$ \\
\hline C5-C6 & $1.359(9)$ \\
\hline C6-C7 & $1.467(9)$ \\
\hline C5-C6ii & $3.803(7)$ \\
\hline C7-O1 & $1.233(6)$ \\
\hline C4'-C5' & $1.43(1)$ \\
\hline C5'-C6' & $1.36(2)$ \\
\hline C6'-C7' & $1.46(1)$ \\
\hline C5'-C6'iii & $3.21(2)$ \\
\hline C7'-O1' & $1.225(6)$ \\
\hline
\end{tabular}

Symmetry codes: (ii) $2-x, 1-y,-z$; (iii) $1-x,-y, 1-z$.

Table 6: Some important angles $\left({ }^{\circ}\right)$ of $\mathrm{C}_{12} \mathrm{H}_{10} \mathrm{O}_{2} \mathrm{~S}$.

\begin{tabular}{|c|c|}
\hline atoms & angle \\
\hline $\mathrm{C} 5 \mathrm{C} 6 \mathrm{C} 7$ & $120.7(5)$ \\
\hline $\mathrm{C} 4 \mathrm{C} 5 \mathrm{C} 6 \mathrm{C} 7$ & $179.9(6)$ \\
\hline $\mathrm{C} 1 \mathrm{~S} 1 \mathrm{C} 4 \mathrm{C} 5$ & $178.3(5)$ \\
\hline $\mathrm{C} 11 \mathrm{~S} 2 \mathrm{C} 8 \mathrm{C} 7$ & $179.7(5)$ \\
\hline $\mathrm{C}^{\prime}{ }^{\prime} 6^{\prime} \mathrm{C} 7^{\prime}$ & $119.3(8)$ \\
\hline $\mathrm{C}^{\prime}{ }^{\prime} 5^{\prime} \mathrm{C} 6^{\prime} \mathrm{C} 7^{\prime}$ & $168.5(8)$ \\
\hline C1'S1'C4'C5' & $176.5(7)$ \\
\hline C11'S'2C8'C7' & $179.3(5)$ \\
\hline
\end{tabular}

During the refinement of the structure, evidence of an unexpected in situ, radiation-induced dimerization reaction was apparent, with the starting and final products coexisting in a topotactic fashion. This kind of reaction have been studied by different research group in similar compound as the cinnamic acid derivates. ${ }^{16}$ In this reaction kinds, the spatial disposition (parallel) of the molecules and the distance between them, is fundamental for the dimerization phenomenon. ${ }^{17}$ The scheme 2 shows the dimerization reaction. 'The X-ray radiation would undergo interactions with the matter and generate ultraviolet photons. This radiation would cause the dimerization phenomenon.

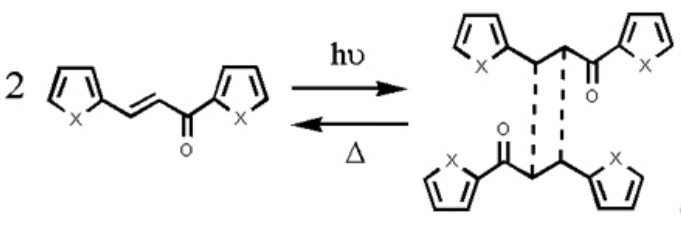

We have done the infra-red spectrum with the hope of observed any change on the structure of the compounds due to ultra-violet radiation. In general the frequencies of the bands in these conjugated compounds are smaller than simple systems. This is due to minor bond order. The infra-red spectrum before the UV radiation is similar to infra-red spectrum after radiation.

This phenomenon is a [2+2] photodimerization homogenous and return thermally to a pure crystal of the monomers. It is the reason why is not possible to detect the structure changes by infra-red analysis.

\section{CONCLUSIONS}

A new heterocyclic system was synthesized, 1-(2-thienyl)-3-(3-methyl2-thienyl)-propenone, $\left(\mathrm{C}_{12} \mathrm{H}_{10} \mathrm{OS}_{2}\right)$ and their crystal structure was established. The structure presents intermolecular interactions of varied type $(\mathrm{C}-\mathrm{H} \cdots \mathrm{O}$, $\mathrm{C}-\mathrm{H} \cdots \pi, \pi \cdots \pi)$ and strength. The anomalies detected in the refinement process, however, provided evidence that an unexpected in-situ, radiationinduced dimerization reaction had taken place during data collection, with the starting and final products coexisting in a topotactic fashion.

\section{ACKNOWLEDGEMENTS}

We acknowledge funding by project DIUC 208.021.026-1.0 and 209.021.028-1.0; Chilean-French Laboratoire International Associé (LIA MIF 836). Dr. Thierry Roisnel of Centre de Diffractométrie X of Université de Rennes 1 "Sciences Chimiques de Rennes" by diffraction experiences. We also acknowledge the Spanish Research Council (CSIC) for providing us with a free-of-charge license to the CSD system. ${ }^{18}$

\section{REFERENCES}

1 Lee, C., Kim, K. J. \& Rhee, S. B. Synth. Met. 69, 295-296, (1995).

2 Diaz, F. R., del Valle, M. A. \& Brovelli, F. J. Applied Polymer Science. 89, 1614-1621, (2003).

3 Nguyen, T. P., Molinie, Handbook of Advanced Electronic and Photonic. P. \& Destruel, P. (2000).

4 Brovelli, F., Del Valle, M. A., Díaz, F. R. \& Bernede, J. C. Bol. Soc. Chil. Quim. 46, 319, (2001).

5 Brovelli, F., Bernede, J. C., Marsillac, S., Díaz, F. R., del Valle, M. A. \& Beaudouin, C. J. Applied Polymer Sci. 86, 1128-1137, (2002).

6 Brovelli, F., Rivas, B. L. \& Basaez, L. J. Chil. Chem. Soc, 48, 135-140, (2003).

7 Distefano, G., Jones, D., Guerra, M., Favaretto, I., Modelli, A. \& Mengoli, G. J. Phys. Chem. 95, 9746-9753, (1991).

8 Cheng, C. L., John, I. G., Ritchie, G. L. D., Gore, P. H. \& Farell, L. J. Chem. Soc. Perkin Trans 2, 744, (1975).

9 Benassi, R., Folli, U., Iarossi, D., Mussatti, A., Nardelli, M., Schenetti, L. \& Taddei, F. J. Chem. Soc. Perkin. Trans 2, 1851, (1987).

10 D. Contreras, Y. Moreno, C. Soto, M. Saavedra, F. Brovelli, R. Baggio. J. Chil. Chem. Soc. 54, 289-291 (2009)

11 Y. Moreno, F. Brovelli, M. Dahrouch, R. Baggio, L. Moreno, J. Chil. Chem. Soc. 55, 331-333, (2010)

12 Bruker (2001). SHELXTL-NT. Bruker AXS Inc., Madison, Wisconsin, USA.

13 Bruker (2006). SMART, SAINT. Bruker AXS Inc., Madison, Wisconsin, USA.

14 Sheldrick, G. M. (1997). SHELXS97 and SHELXL97. University of Göttingen, Germany.

15 Sheldrick, G. M. (2002). SADABS Bruker AXS Inc., Madison, Wisconsin, USA.

16 Murthy, G.S., Ramamurthy, V. Venkatesan K., Acta Crystallographica Section C-Crystal Structure Communications, 44, 307-311, (1988).

17 Ramamurthy,V., Venkatesan, K. Chem. Rev., 87, 433-481,(1987).

18 Allen, F. H. Acta Cryst. B58, 380-388, (2002). 